\title{
Correlación en triatlón masculino entre fases y resultado final en los JJOO de Sídney 2000 Correlation between phases and final result in Men's triathlon competition at the Olympic Games in Sydney 2000 \\ Andrés B. Fernández-Revelles \\ Universidad de Granada (España)
}

Resumen. Introducción: El triatlón es un deporte que se compone de la combinación de tres fases de competición seguidas: nado, bicicleta, carrera y transiciones. La modalidad de triatlón más extendida es la distancia olímpica, y es utilizado en los Juegos Olímpicos (JJOO), se compone de nado 1.5 km, bicicleta $40 \mathrm{~km}$ y carrera $10 \mathrm{~km}$. Objetivos: Determinar la influencia de la duración y orden de las fases con el resultado final de la prueba, y entre fases en el triatlón masculino en los JJOO de Sídney 2000. Material y método: Se analizaron los datos de los 48 deportistas que finalizaron el triatlón masculino en los JJOO de Sídney 2000. Realizando el ranking de cada fase y resultado final para determinar mediante correlación de Spearman la relación entre ellos. Resultados y conclusiones: El resultado final del triatlón no viene determinado ni por la duración de las fases ni por el orden de estas. La fase Run es la que más influye en el resultado final de la prueba en el triatlón con solo un $30.07 \%$ de duración y con una correlación de $r_{s}=.927, p=000$, y la fase Bike es la que menos influye con una correlación de $r=.218, p=1.495$, a pesar de ser la de mayor duración $53.15 \%$.

Palabras clave: Triatlón olímpico, Juegos Olímpicos, fases, reglas de competición, reglas del juego, pruebas combinadas.

Abstract. Introduction: triathlon is a sport consisting of a combination of three consecutive phases of competition: swimming, cycling, running, and the transitions between them. The most widespread modality is the Olympic distance triathlon, used in Olympic Games (OG). This modality comprises of 1.5-km swimming, 40-km cycling, and 10-km running. Aims: to determine the relation between duration and order of the phases and final result, as well as between phases, of the men's Olympic distance triathlon competition during Sydney 2000 OG. Material and Methods: Data from 48 athletes who finished the men's triathlon competition at the Sydney 2000 OG was analysed. Spearmen's correlations were used to establish the relation between athletes' ranking at each phase and their final result. Results and conclusions: The final result of the triathlon competition analysed does not significantly correlate with either duration and order of the phases. The Running phase is the most influential for the overall result of the race as its duration constitutes only $30.07 \%$ of the total, with a correlation index of $r_{s}=.927(p=.001)$, whereas the cycling phase is the least influence $\left(r_{s}=0.218\right.$, $p=1.495$ ) despite being the longest (53.15\% of the total duration).

Key words: Olympic triathlon, Olympic Games, phases, competition rules, game’s rules, combined events.

\section{Introducción}

El triatlón es un deporte que se compone de la combinación de tres fases o segmentos de competición seguidas: nado, bicicleta y carrera, a los que se les suman las correspondientes transiciones entre fases, obteniéndose así el resultado final de la prueba (ITU International Triathlon Union, 2014).

En cada competición se establece un orden o posición en función del tiempo de llegada al finalizar las fases siendo el primero en esa clasificación el ganador de ésta. A partir de este momento al orden, o clasificación que establece el orden de llegada de una fase o de la prueba completa la llamaremos ranking. Cuando una competición de triatlón forma parte de una serie de competiciones a modo de liga o campeonato, las reglas de la competición establecen una puntuación proporcionada en función del ranking en el que acaba cada triatleta cada una de las competiciones. Así gana esa liga o campeonato el que obtenga mayor puntuación en función de los puntos obtenidos por las posiciones ocupadas en los diferentes rankings.

Existen diferentes tipos de triatlones en función de la edad, distancias que se recorren, etc... El tipo de triatlón más extendido y de mayor relevancia es el que tiene distancia olímpica, y que es utilizado en los Juegos Olímpicos (JJOO), que comprende las siguientes fases $1.5 \mathrm{~km}$ nadando (Swim), transición 1, entre natación y bicicleta (T1: Transition 1), 40 km en bicicleta (Bike), transición 2 entre bicicleta y carrera a pie (T2: Transition 2), y $10 \mathrm{~km}$ de carrera a pie (Run), la suma de los tiempos realizados en estas fases da lugar al tiempo total de la prueba (Total Time) que es el resultado con el que se establece la clasificación final de la prueba (ITU International Triathlon Union, 2014). A partir de este momento llamaremos a la suma de las dos fases de transición, Transitions; a la fase de nado Swim; a la fase de bicicleta, Bike; a la carrera, Run; y al resultado final Total Time.

En 1994se aprobó que el triatlón en su modalidad olímpica formase parte de los JJOO tanto en categoría masculina como en categoría

Fecha recepción: 14-10-16. Fecha de aceptación: 10-02-17 Andrés B. Fernández-Revelles abfr@ugr.es femenina, y así ocurrió en los JJOO de Sídney del año 2000 (ITU International Triathlon Union, 2014).

Las reglas del juego que determinan la competición de triatlón establecen para el triatlón olímpico de élite una distancia determinada para cada fase, pero el tiempo empleado en cada fase puede ser diferente de una competición a otra en función de las características del recorrido puesto que se desarrolla al aire libre con unas condiciones que pueden ser cambiantes, en ningún caso el orden de las fases se cambia siendo siempre el mismo.

Podríamos pensar que con unas reglas de juego iguales a todos los competidores las posibilidades de obtener la victoria desde el punto de vista de la estructura de la competición son iguales para todos, pero puede ocurrir que por los elementos y mecanismos que entren en juego en cada competición puedan concurrir circunstancias, como intensidad de esa competición que se adapten mejor al perfil de cada deportista (Zapico, Benito, Diaz, Ruiz, \& Calderon, 2014). De este modo pequeños cambios en los elementos pueden hacer que se beneficie a unos competidores frente otros al igual que ocurre cuando se realizan cambios en los elementos de un deporte o juego para adaptarlos a una población determinada (Méndez-Giménez \& Fernández-Río, 2011).

Por esta razón vemos importante realizar un análisis de las distintas competiciones de triatlón en citas olímpicas, empezando por la primera que se realizó en Sídney 2000, para contrastar de una forma empírica que, no existiendo cambios en las reglas del juego, al concurrir diferentes elementos del juego en cada competición pueda existir un beneficio hacia un perfil de participante (Nacke, Bateman, \& Mandryk, 2011; Rivas et al., 2015; Tondello et al., 2016).

El triatlón tiene grandes demandas fisiológicas, de fuerza (Bentley, Wilson, Davie, \& Zhou, 1998), potencia (Bernard et al., 2009), biomecánicas, (Le Meur et al., 2013), cognitivas, psicológicas (Jaenes Sánchez, Peñaloza Gómez, Navarrete Dueñas, \& Bohórquez GómezMillán, 2012; Leruite, Morente-Sánchez, Martos, Girela, \& Zabala, 2016) y entrenamiento muy exigentes y específicas sobre todo en resistencia que es importante conocer debido a las características tan diferentes de cada una de las fases y su relación con el resultado final (Bentley, Millet, Vleck, \& McNaughton, 2002; Ramos-Campo, Martínez, Esteban, Rubio-Arias, \& Jiménez, 2016). 
En el rendimiento de los deportistas pueden influir las condiciones del recorrido si por ejemplo se realiza en zonas tropicales (Hue, 2011), o el calor corporal generado durante la prueba (Kerr, Trappe, Starling, \& Trappe, 1998), por el frio (Dallam, Jonas, \& Miller, 2005) aspectos que han de ir directamente relacionados con una adecuada nutrición (Jeukendrup, Jentjens, \& Moseley, 2005) e hidratación (Noakes, 2007) durante la prueba.

Hay que unir la estrategia de cada triatleta en función de sus características y posibilidades en cada fase (Abbiss \& Laursen, 2008; Atkinson \& Brunskill, 2000; Hausswirth \& Brisswalter, 2008; Johnson et al., 2015; Le Meur et al., 2009) además de las diferentes variables que pueden surgir durante la competición como el drafting (Etxebarria, D’Auria, Anson, Pyne, \& Ferguson, 2014; Hausswirth \& Brisswalter, 2008; Millet \& Vleck, 2000; Peeling \& Landers, 2009).

En función de las características de cada deportista o el tipo de entrenamiento utilizan diferentes estrategias entre fases o en cada una de las fases para optimizar el rendimiento (Hausswirth \& Brisswalter, 2008; Hausswirth, Le Meur, Bieuzen, Brisswalter, \& Bernard, 2010; Le Meur et al., 2011; Peeling \& Landers, 2009). Así por ejemplo cuando un triatleta detecta cansancio en una fase del triatlón en la siguiente fase economiza la energía con el fin de no llegar a estar exhausto (Bonacci, Vleck, Saunders, Blanch, \& Vicenzino, 2013).

Así del conocimiento de la duración e intensidad de cada una de las fases como de la relación de cada fase con el resultado final se pueden extraer consecuencias aplicables tanto a la planificación del entrenamiento como a la estrategia de entrenamiento y desarrollo de la prueba.

\section{Objetivo}

El objetivo de este estudio es determinar la influencia de la duración, orden de las fases, y qué fase tiene más influencia con el resultado final de la prueba en el triatlón masculino en los JJOO de Sídney 2000.

\section{Material y método}

\section{Obtención de datos}

Para la obtención de los datos de este estudio, se siguió el procedimiento utilizado en (Fernandez-Revelles, 2012, 2014) que consiste en descargar de las bases de datos a una hoja de cálculo todos los datos que coinciden con nuestra búsqueda (Fernandez-Revelles, 2013; FernandezRevelles et al., 2009) utilizando las palabras «2000», «man», «triathlon», en este caso recoger los datos oficiales de la competición incluidos en (ITU International Triathlon Union, 2015).

\section{Muestra}

En el presente estudio se utilizó el censo completo de la competición y se desecharon los datos de los atletas que no completaron la competición, siendo 48 los atletas que la completaron (ITU International Triathlon Union, 2015).

El tratamiento de los datos se realizó con una tabla de Excel transformando los datos de tiempos de cada fase y finales a segundos realizando un análisis descriptivo de las variables (tabla 1).

\begin{tabular}{lcccc} 
Tabla 1 \\
Descriptivos de los datos recogidos \\
\hline \multicolumn{5}{l}{$N$} \\
\hline Swim 2000 Man & 48 & 1079.44 & 23.966 & 574.379 \\
Bike 2000 Man & 48 & 3553.96 & 87.192 & 7602.424 \\
Run 2000 Man & 48 & 2010.63 & 117.001 & 13689.346 \\
Transitions 2000 Man & 48 & 42.58 & 4.297 & 18.461 \\
Total Time 2000 Man & 48 & 6689.98 & 161.348 & 26033.127 \\
\hline
\end{tabular}

Denominamos a estas seis variables con su nomenclatura en inglés, con los resultados de cada fase, Swim, Transition 1, Bike, Transition 2, Run y Total Time, las resumimos en cinco variables sumando Transition 1 y 2, debido al poco tiempo empleado en cada una. Quedando así las cinco variables Swim, Bike, Run, Transitions y Total Time.

A partir de estas cinco variables utilizando las fórmulas de Excel jerarquizamos por tiempos cada una de las variables y obtenemos otras cinco variables con los rankings de cada una de las variables, siguiendo la metodología anteriormente utilizada (Fernandez-Revelles, 2012, 2014).

\section{Análisis estadístico}

Las variables son importadas a SPSS 22 con el que realizamos los siguientes análisis:

Suma de los tiempos de las variables para hallar los porcentajes de tiempo empleado en cada fase (figura 1 ).

Correlación de Spearman entre rankings de fases y ranking final para determinar la relación de cada fase con el resultado final, (tabla 2).

Correlación de Spearman entre los rankings de las fases para determinar la relación entre ellos, (tabla 3).

Se halló además el parámetro $Z$ de la regresión lineal y el coeficiente de determinación lineal $R^{2}$. Además de realizar diferentes figuras para ilustrar el estudio.

\section{Resultados}

Los resultados muestran, (figuras 1 y 2), como más del 50\% del tiempo empleado en la prueba se ha utilizado en realizar la fase Bike.

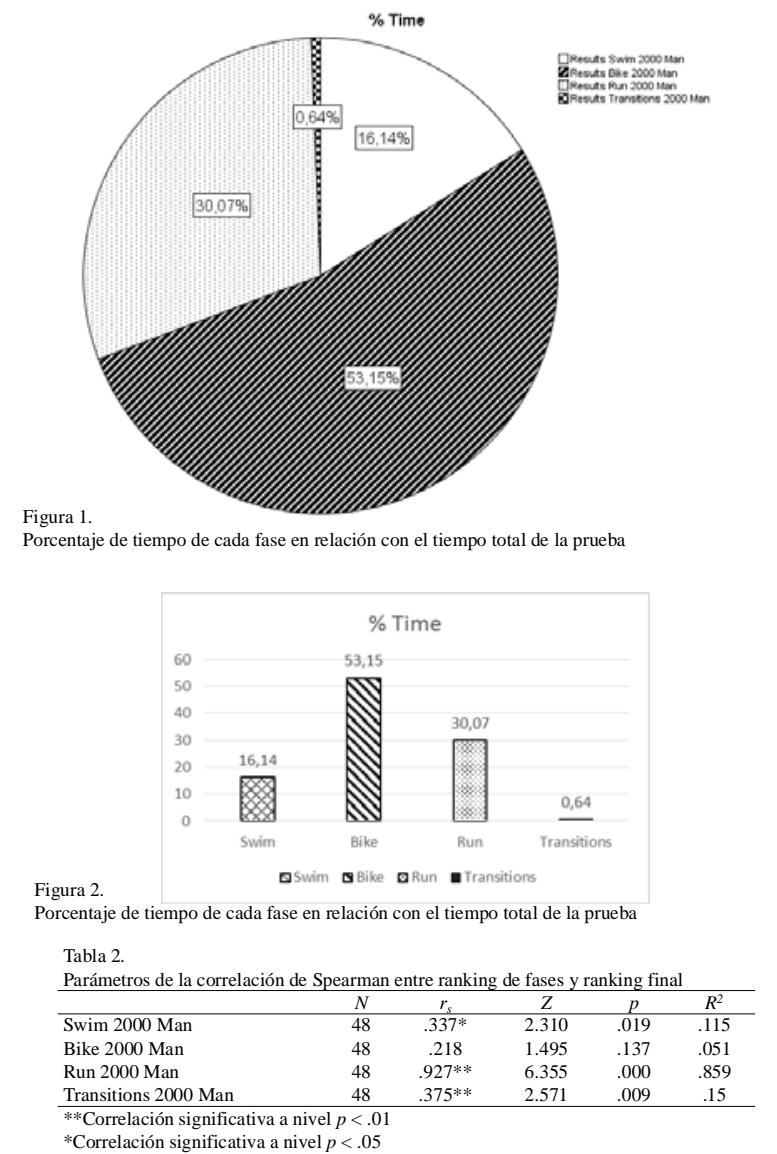

Los resultados de las correlaciones de Spearman, (tabla 2), indican una correlación muy alta entre la fase Run y Total Time con un $r_{\mathrm{s}}=.927$, $p=.000$, y coeficiente de determinación lineal $R^{2}=.859$.

Los resultados de las correlaciones de Spearman, (tabla 3), muestran que no existe correlación entre las fases Swim-Run, SwimTransitions, Bike-Run y Bike-Transitions. Por el contrario, si existe una correlación moderada entre las fases Run-Trasnsitions, (figura 8), con un $r=.391, p=.006$, y coeficiente de determinación lineal $R^{2}=.153$; y también existe una correlación moderada entre las fases Swim-Bike, en este caso la correlación es inversa, (figura 7), con un $r_{s}=.480, p=.001$

Tabla 3.

\begin{tabular}{lccccc}
\multicolumn{7}{l}{ Parámetros de la correlación de Spearman entre ranking y fases } \\
\hline \multicolumn{7}{c}{$N$} & $r_{s}$ & $Z$ & $p$ & $R^{2}$ \\
\hline Swim-Bike 2000 Man & 48 & $-.480^{* *}$ & -3.291 & .001 & .230 \\
Swim-Run 2000 Man & 48 & .284 & 1.947 & .051 & .081 \\
Swim-Transitions 2000 Man & 48 & -.002 & -.014 & .990 & .000 \\
Bike-Run 2000 Man & 48 & -.006 & -.041 & .970 & .000 \\
Bike-Transitions 200 Man & 48 & .041 & .281 & .781 & .002 \\
Run-Transitions 2000 Man & 48 & $.391^{* *}$ & 2.681 & .006 & .153 \\
\hline **Correlación significativa a nivel $p<.01$ & & & & \\
*Correlación significativa a nivel $p<.05$ & & & &
\end{tabular}




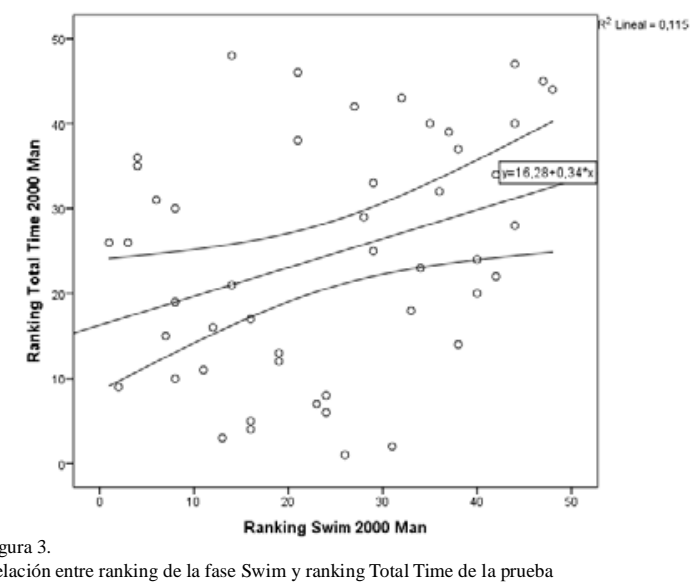

Relación entre ranking de la fase Swim y ranking Total Time de la prueba

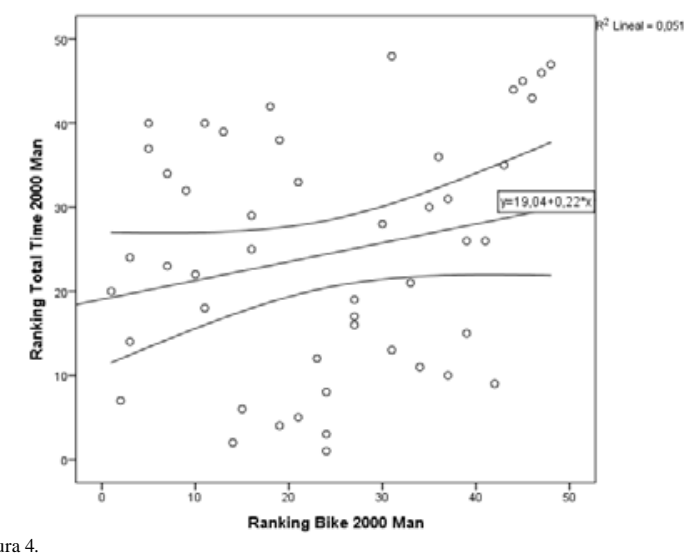

Relación entre ranking de la fase Bike y ranking Total Time de la prueba

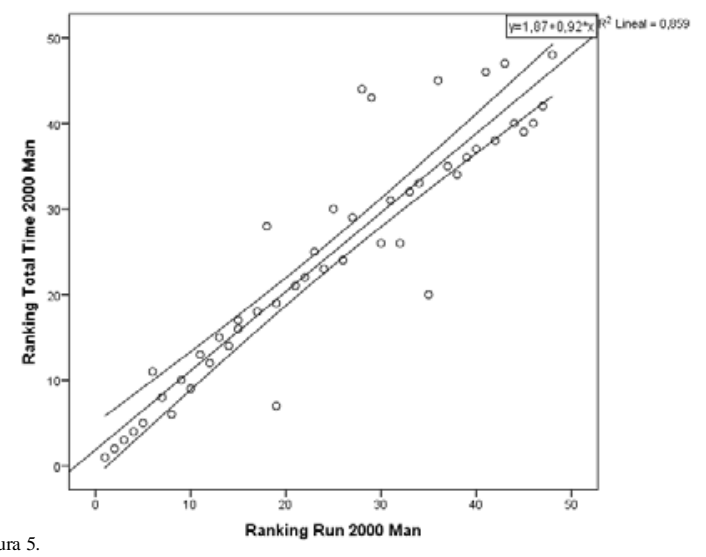

Relación entre ranking de la fase Run y ranking Total Time de la prueba

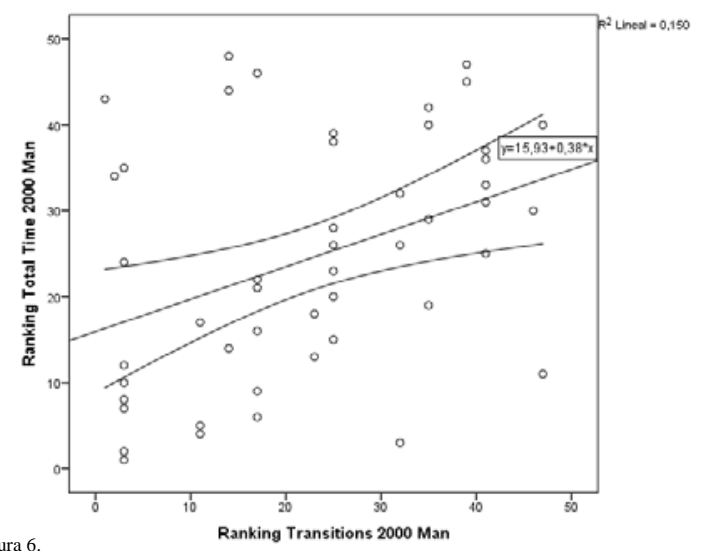

Figura 6 .

Relación entre ranking de la fase Transitions y ranking Total Time de la prueba

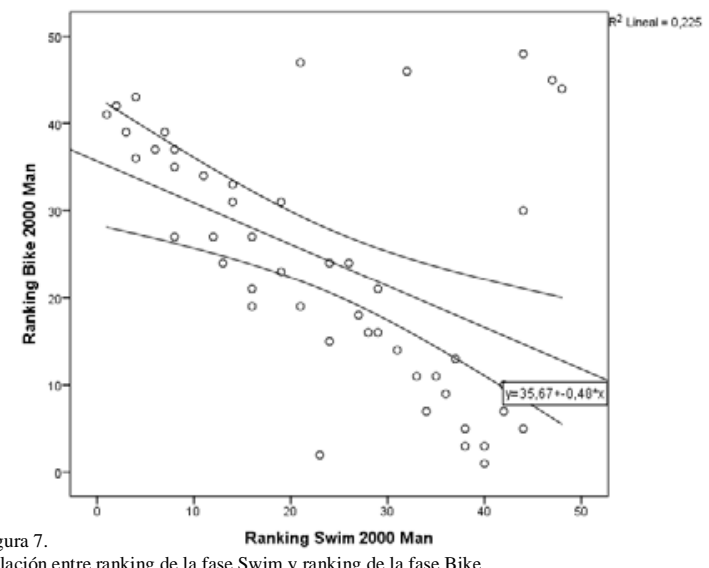

Relación entre ranking de la fase Swim y ranking de la fase Bike

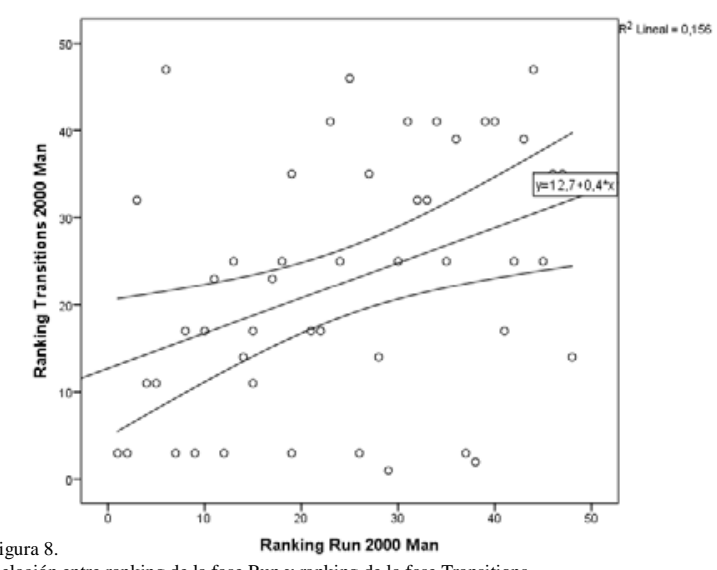

Relación entre ranking de la fase Run y ranking de la fase Transitions

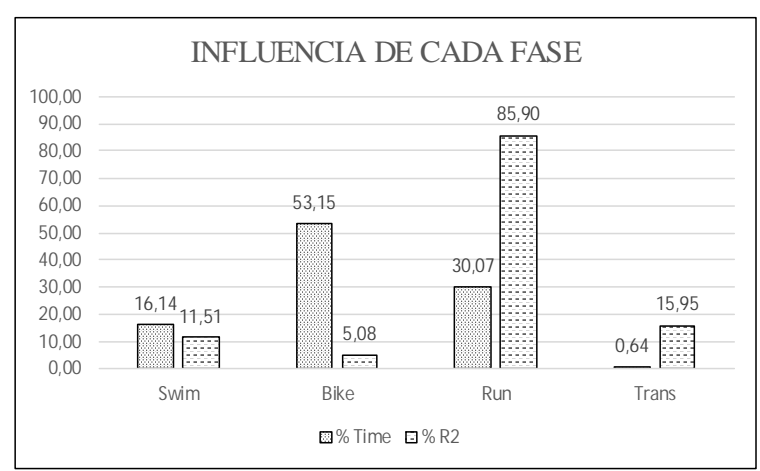

Figura 9

Influencia de cada fase en la prueba en función del porcentaje de tiempo empleado en cada fase y del porcentaje de ranking final determinado por cada fase en función del coeficiente de determinación lineal $R^{2}$.

y coeficiente de determinación lineal $R^{2}=.230$.

Se muestra un resumen descriptivo de los datos a partir de los cuales se realiza el ranking de cada variable (tabla 1). La relación del ranking de cada fase con el ranking Total Time se puede visualizar (figura 9). Muestra la influencia de cada fase de la prueba con respecto al ranking Total Time en función del porcentaje de tiempo empleado. La relación entre estas variables queda determinada por el coeficiente de determinación lineal $R^{2}$.

Hay que indicar que para la correcta interpretación (figura 9) hay que tener en cuenta que los porcentajes de tiempo empleados en cada fase de la prueba suman un total del $100 \%$ del Total Time empleado en la prueba, y que sin embargo la suma de los coeficientes de determinación lineal $R^{2}$ en cada fase podrían ser del $100 \%$ si el ranking de esa fase fuese igual al ranking Total Time, por consiguiente los porcentajes calculados a través de los coeficientes de determinación lineal $R^{2}$ de cada 
fase pueden solaparse, aunque indican claramente el porcentaje que el ranking de esa fase predice el ranking Total Time como resultado final.

\section{Discusión}

Los resultados indican que el tiempo empleado en cada fase del triatlón de los JJOO de Sídney 2000, es muy diferente, y que a mayor tiempo empleado en una fase no existe mayor influencia del ranking de esa fase con el ranking del resultado final (figura 9).

Al ser el triatlón una competición cuyo resultado final se compone de la suma de las fases Swim, Bike, Run y las Transitions entre fases se podría esperar que la fase en la que se emplea más tiempo tuviera mayor influencia en el ranking Total Time, sin embargo, los datos muestran que la fase del triatlón en la que se emplea más tiempo es Bike 53.15\%, (figuras 1, 2 y 9), no existiendo correlación entre el ranking de la fase Bike y el ranking Total Time como muestran (tabla 2) y (figura 4).

La fase Run es la fase que más influencia tiene en este triatlón en relación con el ranking Total Time, (tabla 2) y (figura 5) que indican una correlación muy alta. Significa que el 85.9 del ranking del Total Time viene determinado por el ranking de la fase Run, a pesar de ocupar solamente el 30.07\% de tiempo del Total Time.

Como la fase Run es la última fase podría parecer que el orden en el que se realizan las fases influye más en el ranking Total Time, así las últimas fases influirían más frente a las primeras. En este triatlón puede parecer así puesto que la primera fase Swim muestra una baja relación entre ranking Swim y ranking Total Time, (figura 3), inferior a la relación del ranking de la fase Run con el ranking Total Time.

Con el ranking de la fase Swim que es la primera podríamos conocer un $11.5 \%$ del ranking Total Time o clasificación final. La relación entre ranking Swim y ranking Total Time es mayor que la relación ranking Bike con ranking Total Time, así por tanto el orden de la fase queda descartado en este triatlón. Además, la relación ranking Swim y ranking Total Time, (figura 3, 4, y 9), es mayor que la relación que tiene ranking Bike a pesar de que la fase Swim sólo emplea un 16.14 del Total Time frente al 53.15\% de la fase Bike.

Como curiosidad en este triatlón en la fase Transitions con sólo un .64\% del tiempo de la prueba, tiene una correlación la fase ranking de Transitions con el ranking Total Time entre baja y moderada, (tabla 3), (figura 6). Lo que se podría interpretar como que un 15\% del ranking del Total Time viene determinado por el ranking Transitions. Puede tener una interpretación relacionada con el entrenamiento y rendimiento de la prueba indicando que con una relación moderada que los que han entrenado la fase Transitions y han obtenido mejor rendimiento en la fase Transitions son los que obtienen mejor rendimiento al final de la prueba.

En el análisis realizado también podemos observar la relación entre los rankings de las fases. El resultado que más destaca es la relación inversa y moderada entre ranking Swim y ranking Bike con un un $r_{s}=$ $.480, p=.001$, y coeficiente de determinación lineal $R^{2}=.230$, (tabla 3), (figura 7), que podría interpretarse como una influencia negativa del 23\% del ranking de la fase Swim con el ranking de la fase Bike, que podría estar determinado por el cansancio acumulado en la anterior fase.

\section{Sugerencias para futuros trabajos}

Con este trabajo se abre un abanico de futuras investigaciones que analicen las diferentes modalidades de triatlón en función de la relación de las fases con el resultado final, dado que el tiempo empleado en el ranking en cada fase no parece ser proporcional al ranking del resultado final.

En principio habría que partir analizando los triatlones de los JJOO para determinar si se repiten las pautas observadas en este trabajo.

Posteriormente trasladar el análisis a las diferentes modalidades de triatlón en función de la distancia, además de por categorías de edad y sexo.

También se podría contemplar en función de los resultados obtenidos una modificación de las reglas del juego en este caso en la competición del triatlón para que cada una de las fases tuviese similar incidencia en el resultado final, o que existiese una proporcionalidad en el tiempo empleado en cada fase con el resultado final.

Siguiendo con la idea de la modificación de las reglas del juego también se podrían proponer coeficientes correctores en función de los resultados de cada fase con el resultado final.

\section{Conclusión}

El resultado final del triatlón masculino en los JJOO de Sídney 2000 no viene determinado ni por la duración de las fases ni por el orden de estas, siendo la fase Run la que más influye al resultado final de la prueba en el triatlón masculino en los JJOO de Sídney 2000, y la fase Bike es la que menos influye a pesar de ser la de mayor duración.

\section{Referencias}

Abbiss, C. R., \& Laursen, P. B. (2008). Describing and understanding pacing strategies during athletic competition. Sports Medicine,38(3), 239-252.

Atkinson, G., \& Brunskill,A. (2000). Pacing strategies during a cycling time trial with simulated headwinds and tailwinds. Ergonomics, 43(10), 1449-1460. doi: 10.1080/001401300750003899

Bentley, D. J., Millet, G. P., Vleck, V. E., \& McNaughton, L. R. (2002). Specific aspects of contemporary triathlon - Implications for physiological analysis and performance. Sports Medicine, 32(6), 345-359. doi: 10.2165/00007256-200232060-00001

Bentley, D. J., Wilson, G. J., Davie, A. J., \& Zhou, S. (1998). Correlations between peak power output, muscular strength and cycle time trial performance in triathletes. Journal of Sports Medicine and Physical Fitness, 38(3), 201-207.

Bernard, T., Hausswirth, C., Le Meur, Y., Bignet, F., Dorel, S., \& Brisswalter, J. (2009). Distribution of Power Output during the Cycling Stage of a Triathlon World Cup. Medicine and Science in Sports and Exercise, 41(6), 1296-1302. doi: 10.1249/ MSS.0b013e318195a233

Bonacci, J., Vleck, V., Saunders, P. U., Blanch, P., \& Vicenzino, B. (2013). Rating of perceived exertion during cycling is associated with subsequent running economy in triathletes. Journal of Science and Medicine in Sport, 16(1), 49-53. doi: 10.1016/ j.jsams.2012.04.002

Dallam, G. M., Jonas, S., \& Miller, T. K. (2005). Medical considerations in triathlon competition-Recommendations for triathlon organisers, competitors and coaches. Sports Medicine, 35(2), 143-161. doi: 10.2165/00007256-200535020-00004

Etxebarria, N., D’Auria, S., Anson, J. M., Pyne, D. B., \& Ferguson, R. A. (2014). Variability in Power Output During Cycling in International Olympic-Distance Triathlon. International Journal of Sports Physiology and Performance, 9(4), 732-734. doi: 10.1123/ ijspp.2013-0303

Fernandez-Revelles, A. B. (2012).ABFR-Index: correlación entre producción científica y Juegos Olímpicos 2008. Habilidad Motriz,38, 51-57.

Fernandez-Revelles, A. B. (2013). Modelo matemático de ley de potencias aplicado al maratón. Habilidad Motriz, 41, 12-20.

Fernandez-Revelles, A. B. (2014). ABFR-Index: correlation between «soccer» scientific production and ranking. Revista Internacional De Medicina Y Ciencias De La Actividad Fisica Y Del Deporte, 14(56), 705-718. doi: Http://cdeporte.rediris.es/revista/revista56/ artABFR506.htm

Fernandez-Revelles, A. B., Robles, A., Dafos, J., Soto, V. M., PerezCortes, A. J., Latorre, P., . . Romero, C. (2009). Physical activity: Evaluation of research in Spain. Gaceta sanitaria, 23, 204-204.

Hausswirth, C., \& Brisswalter, J. (2008). Strategies for Improving Performance in Long Duration Events Olympic Distance Triathlon. Sports Medicine, 38(11), 881-891. doi: 10.2165/00007256200838110-00001

Hausswirth, C., Le Meur, Y., Bieuzen, F., Brisswalter, J., \& Bernard, T. (2010). Pacing strategy during the initial phase of the run in triathlon: 
influence on overall performance. European Journal of Applied Physiology, 108(6), 1115-1123. doi: 10.1007/s00421-009-1322-0 Hue, O. (2011). The Challenge of Performing Aerobic Exercise in Tropical Environments: Applied Knowledge and Perspectives. International Journal of Sports Physiology and Performance, 6(4), 443-454.

ITU International Triathlon Union. (2014, December-2014). ITU Competition Rules. Recuperado dehttp://www.triathlon.org/about/ documents

ITU International Triathlon Union. (2015). ITU Competition Results. Recuperado de http://www.triathlon.org/results

Jaenes Sánchez, J. C., Peñaloza Gómez, R., Navarrete Dueñas, K. G., \& Bohórquez Gómez-Millán, M. R. (2012). Ansiedad y autoconfianza precompetitiva en triatletas. Revista iberoamericana de psicología del ejercicio y el deporte, 7(1), 113-124.

Jeukendrup, A. E., Jentjens, R., \& Moseley, L. (2005). Nutritional considerations in triathlon. Sports Medicine, 35(2), 163-181. doi: 10.2165/00007256-200535020-00005

Johnson, E. C., Pryor, J. L., Casa, D. J., Belval, L. N., Vance, J. S., DeMartini, J. K., ... Armstrong, L. E. (2015). Bike and run pacing on downhill segments predict Ironman triathlon relative success. Journal of Science and Medicine in Sport, 18(1),82-87. doi:10.1016/ j.jsams.2013.12.001

Kerr, C. G, Trappe, T. A., Starling, R. D., \& Trappe, S. W. (1998). Hyperthermia during Olympic triathlon: influence of body heat storage during the swimming stage. Medicine and Science in Sports and Exercise, 30(1), 99-104. doi: 10.1097/00005768-19980100000014

Le Meur, Y., Bernard, T., Dorel, S., Abbiss, C. R., Honnorat, G., Brisswalter, J., \& Hausswirth, C. (2011). Relationships Between Triathlon Performance and Pacing Strategy During the Run in an International Competition. International Journal of Sports Physiology and Performance, 6(2), 183-194.

Le Meur, Y., Hausswirth, C., Dorel, S., Bignet, F., Brisswalter, J., \& Bernard, T. (2009). Influence of gender on pacing adopted by elite triathletes during a competition. European Journal of Applied Physiology, 106(4), 535-545. doi: 10.1007/s00421-009-1043-4

Le Meur, Y., Hausswirth, C., Natta, F., Couturier, A., Bignet, F., \& Vidal, P. P. (2013). A multidisciplinary approach to overreaching detection in endurance trained athletes. Journal of Applied Physiology, 114(3), 411-420. doi: 10.1152/japplphysiol.01254.2012

Leruite, M., Morente-Sánchez, J., Martos, P., Girela, M. J., \& Zabala, M. (2016). Analysis of the Sporting Context of Spanish Female
Competitive Cyclists and Triathletes. Revista Internacional De Medicina Y Ciencias De La Actividad Fisica Y Del Deporte, 16(64), 667-684.

Méndez-Giménez, A., \& Fernández-Río, J. (2011). Análisis y modificación de los juegos y deportes tradicionales para su adecuada aplicación en el ámbito educativo. Retos. Nuevas tendencias en Educación Física, Deporte y Recreación, 19, 54-58.

Millet, G. P., \& Vleck, V. E. (2000). Physiological and biomechanical adaptations to the cycle to run transition in Olympic triathlon: review and practical recommendations for training. British Journal of Sports Medicine, 34(5), 384-390. doi: 10.1136/bjsm.34.5.384

Nacke, L. E., Bateman, C., \& Mandryk, R. L. (2011). BrainHex: Preliminary Results from a Neurobiological Gamer Typology Survey. In J. Anacleto, S. Fels, N. Graham, B. Kaparalos, M. S. ElNasr, \& K. Stanley (Eds.), Entertainment Computing - Icec 2011 (Vol. 6972, pp. 288-293). Berlin: Springer-Verlag Berlin.

Noakes, T.D. (2007). Drinking guidelines for exercise: What evidence is there that athletes should drink "'as much as tolerable", "'to replace the weight lost during exercise" or "ad libitum"? Journal of Sports Sciences, 25(7), 781-796. doi: 10.1080/02640410600875036

Peeling, P., \& Landers, G. (2009). Swimming intensity during triathlon: A review of current research and strategies to enhance race performance. Journal of Sports Sciences, 27(10), 1079-1085. doi: 10.1080/ 02640410903081878

Ramos-Campo, D. J., Martínez, F., Esteban, P., Rubio-Arias, J. A., \& Jiménez, J. F. (2016). Intermittent hypoxic training and cycling performance in triathletes. Revista Internacional De Medicina $Y$ Ciencias De La Actividad Fisica Y Del Deporte, 16(61), 139-156. doi: http://dx.doi.org/10.15366/rimcafd2016.61.011

Rivas, L. G., Mielgo-Ayuso, J., Norte-Navarro,A., Cejuela, R., Cabanas, M. D., \& Martinez-Sanz, J. M. (2015). Body composition and somatotype in university triathletes. Nutricion Hospitalaria, 32(2), 799-807. doi: 10.3305/nh.2015.32.2.9142

Tondello, G. F., Wehbe, R. R., Diamond, L., Busch, M., Marczewski, A., Nacke, L. E., \& Acm. (2016). The Gamification User Types Hexad Scale. Chi Play 2016: Proceedings of the 2016 Annual Symposium on Computer-Human Interaction in Play, 229-243. doi: 10.1145/2967934.2968082

Zapico, A. G, Benito, P. J., Diaz, V., Ruiz, J. R., \& Calderon, F. J. (2014). Heart rate profile in highly trained triathletes. Revista Internacional De Medicina Y Ciencias De La Actividad Fisica Y Del Deporte, 14(56), 619-632. doi: Http://cdeporte.rediris.es/revista/ revista56/artperfil505.htm
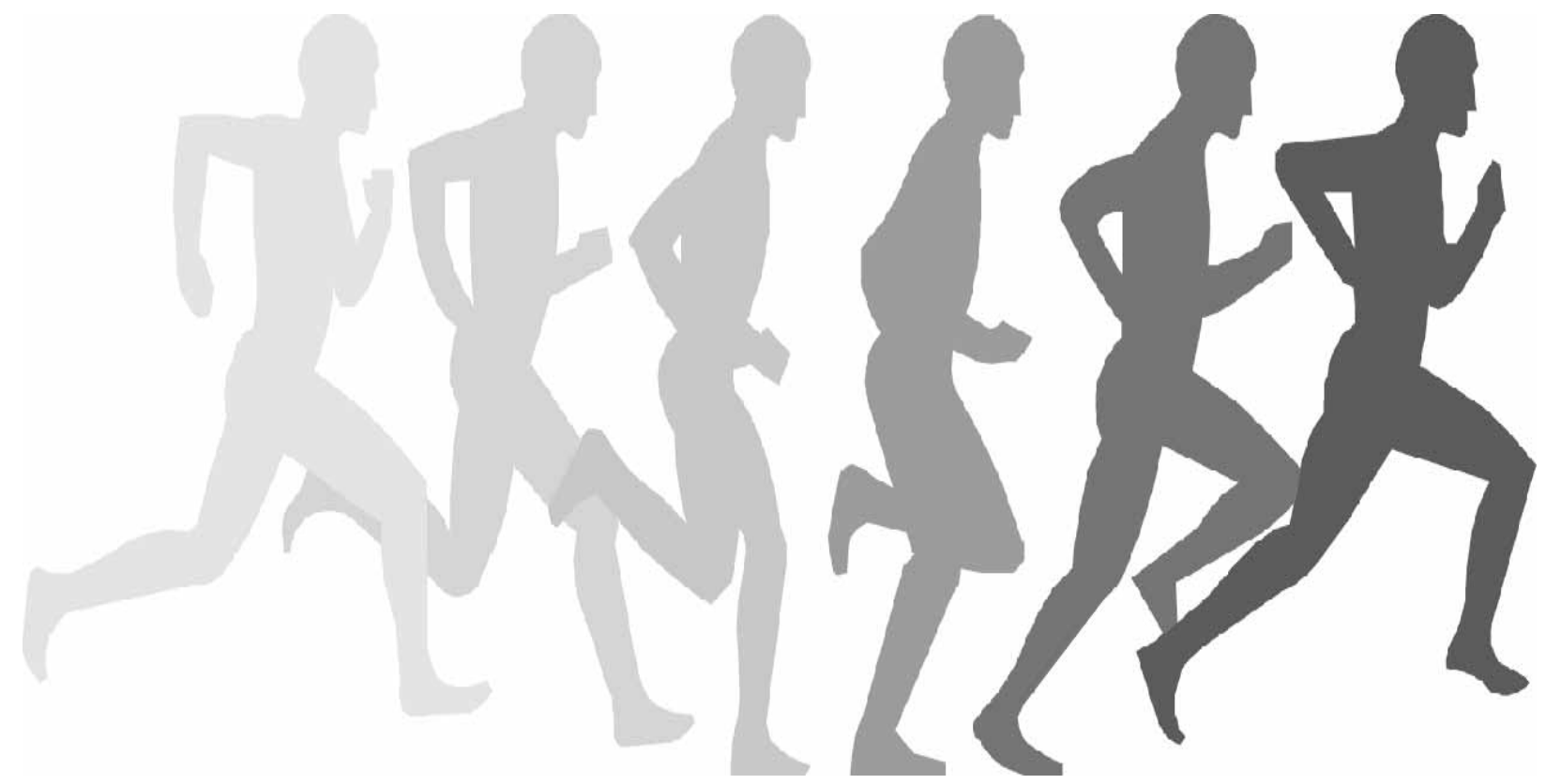